\title{
Arthropod prey vary among orders in their nutrient and exoskeleton content
}

\author{
Jacob Reeves ${ }^{1}$, Samuel Fuhlendorf ${ }^{2}$, Craig Davis ${ }^{2}$, and Shawn Wilder ${ }^{2}$ \\ ${ }^{1}$ Oklahoma State University Stillwater \\ ${ }^{2}$ Oklahoma State University
}

June 23, 2021

\begin{abstract}
Insectivores gain macronutrients and elements from consuming arthropod prey, but must also deal with indigestible components (i.e., exoskeleton) of prey. For example, avian chicks (e.g. northern bobwhites; Colinus virginianus) have limited gut space, and ingesting prey with relatively higher proportions of indigestible components may impact assimilation efficiency, growth, and survival. The ability of insectivores to choose higher quality prey would depend on prey taxa varying consistently in nutritional content. We tested if there were consistent differences among taxonomic orders of arthropod prey in their macronutrient (protein and lipid), elemental (C and $\mathrm{N}$ ), and exoskeleton content. We used northern bobwhite chicks as our focal insectivore and focused on their potential prey as a case study. We also tested the influence of indigestible exoskeleton on the measurement of macronutrient content and the ability of elemental content to predict macronutrients. We found large and consistent variation in macronutrient and elemental content within and between arthropod orders. Some orders had consistently high protein content and low exoskeleton content (i.e., Araneae) and are likely higher quality prey for insectivores. Abundant orders common in the diets of insectivores, like Hymenoptera and Coleoptera, had high exoskeleton content and low protein content. We also found support for the ability of elements to predict macronutrients, and found that metabolizable (i.e. exoskeleton removed) elemental content better predicted macronutrient content. A better understanding of arthropod nutrient content is critical for elucidating the role of spatial and temporal variation in prey communities in shaping the growth and survival of insectivores.
\end{abstract}

\section{Hosted file}

Reeves arthropod nutrient content.pdf available at https://authorea.com/users/421338/ articles/527411-arthropod-prey-vary-among-orders-in-their-nutrient-and-exoskeletoncontent 